\title{
Application of the Fuel-Optimal Energy Management in Design Study of a Parallel Hybrid Electric Vehicle
}

\author{
Afshin Pedram Pourhashemi, ${ }^{1,2}$ S. M. Mehdi Ansarey Movahed, ${ }^{3}$ \\ and Masoud Shariat Panahi ${ }^{3}$ \\ ${ }^{1}$ Department of Mechanical Engineering, Qazvin Azad University, Qazvin 34185-1416, Iran \\ ${ }^{2}$ Iran Test and Research Auto Company (ITRAC), Tehran 13855-155, Iran \\ ${ }^{3}$ School of Mechanical Engineering, University of Tehran, Tehran 11365-4563, Iran \\ Correspondence should be addressed to Afshin Pedram Pourhashemi; apedramm@gmail.com
}

Received 13 June 2014; Revised 16 August 2014; Accepted 20 August 2014; Published 7 October 2014

Academic Editor: Bruno G. Pollet

Copyright (C) 2014 Afshin Pedram Pourhashemi et al. This is an open access article distributed under the Creative Commons Attribution License, which permits unrestricted use, distribution, and reproduction in any medium, provided the original work is properly cited.

\begin{abstract}
In spite of occasional criticism they have attracted, hybrid vehicles (HVs) have been warmly welcomed by industry and academia alike. The key advantages of an HV, including fuel economy and environment friendliness, however, depend greatly on its energy management strategy and the way its design parameters are "tuned." The optimal design and sizing of the HV remain a challenge for the engineering community, due to the variety of criteria and especially dynamic measures related to nature of its working conditions. This paper proposes an optimal design scheme that begins with presenting an energy management strategy based on minimum fuel consumption in finite driving cycle horizon. The strategy utilizes a dynamic programming approach and is consistent with charge sustenance. The sensitivity of the vehicle's performance metrics to multiple design parameters is then studied using a design of experiments (DOE) methodology. The proposed scheme provides the designer with a reliable tool for investigating various design scenarios and achieving the optimal one.
\end{abstract}

\section{Introduction}

Rapidly shrinking fossil fuel resources and growing environmental concerns have motivated a great deal of research on hybrid vehicles. Strict emission standards and rising fuel prices have encouraged manufactures to move away from conventional vehicles. Hybridization of conventional vehicles has proven to be the most efficient short-term solution to the problem. Hybrid vehicles enjoy attractive features such as the ability to shift the operating point of the internal combustion engine (ICE) according to a control plan, the restoration of brake energy, and the ability to switch to a pure electric mode in case of hybrid electric vehicle (HEVs).

Integration of an additional energy source (battery) leads to an extra degree of freedom (DOF), since the propulsion force can be provided either by the ICE or by the electric machine (EM), and thus a suitable energy management strategy (EMS) should be used to control this diversity.
Development of an EMS is an important task in the design of hybrid vehicles and the literature is relatively rich on this topic [1].

The design of an EMS is commonly treated as a dynamic optimization problem where the causality is not a major concern. Furthermore, selection of the proper topology (including transmission) and proper sizes of the power source components (sizing) are treated as extra layers of the optimization problem in recent hybrid propulsion studies [2] (see Figure 1). Those extra layers are mainly concerned with the reduction of fuel consumption and emissions while satisfying drivability constraints. Unfortunately, however, the interaction of those extra layers and the complexity of the problem make it computationally expensive to simultaneously consider all different layers in design studies.

Since the traditional design process cannot reveal the ultimate potential of hybridization, a bilevel strategy is proposed for the selection of optimal hybridization ratio (HR) 


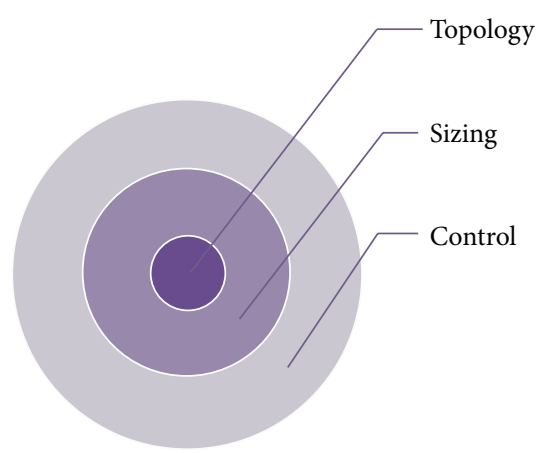

Figure 1: Various levels of the hybrid vehicle design process.

of a parallel HEV in which the inner level is governed by a boundary dynamic programming (DP) scheme and the outer level is addressed full factorial iteration on HRs [3].

In a recently published work, emission has been added to the sizing criteria for the optimal sizing of a diesel $\mathrm{HEV}$ [4]. In another work, the optimal cost and fuel selection for HEV topologies have been investigated using particle swarm optimization and DP [5]. Pontryagin's minimum principles (PMP) have been also used for the optimal control of HEVs in [6]. A switching topology with automated transmission has been considered in order to evaluate the effect of component sizes and transmission technologies on fuel economy [7]. Similar investigations have been made for the optimal sizing of the fuel cell HV in [8].

The EMS is an integrated part of $\mathrm{HV}$ simulation, whether it is traditional rule-based method or offline optimal control. As concluded from the literature, the optimal control method has been favored in many HEV design studies, but that is not the only way to tackle the problem. Among other approaches are the optimization of the parameterized rulebased EMS along with the design variables to solve the sizing problem [9] and a simplified version of the stochastic dynamic programming for the design of fuel cell HVs [10].

1.1. Structure of the Article. This paper introduces a novel optimal energy management strategy with full charge sustenance based on DP. It also evaluates the effects of design variables and drivability constraints on fuel economy.

It is important to use an optimal approach to EMS, because sizing (design) and energy management strategy (control) are inherently coupled, meaning that a different set of component sizes needs the tuning of the traditional heuristic-based energy management strategy. This mutual influence is emphasized in [11] for a combined plant/controller optimization. Unlike the rule-based and heuristic methods, the optimal EMS is capable of seeking the ultimate potential of the efficiency improvements.

This paper is mainly concerned with the sizing problem in the design of parallel HEVs. Therefore, for the elimination of the EMS effects on FC, an optimal algorithm based on DP is introduced and it is modified to fully satisfy the fixed final state constraint (boundary-line DP). Ensuring charge sustenance (equal initial and final states-of-charge) is vital to have a fair comparison between different sizes. The proposed algorithm solves the energy management problem within the framework of a finite-horizon optimal control problem.

Further, design study focuses on the effects made on fuel economy by the power source sizes. Without imposing drivability constraints, a two-dimensional map of the fuel economy will be generated for the feasible pairs of the ICE and EM peak power. The simulation repeated for several driving cycles to demonstrate the effects of driving cycle characteristics on optimal sizing of the power sources, regarding defined acceleration performance.

This paper is organized as follows. Firstly, the powertrain model is described. Using this model, the energy management problem is formulated and the corresponding solution algorithm is described. Based on this algorithm and defining sizing objective, the design study of the HEV is fulfilled and the results of design study are presented in several driving cycle.

\section{Powertrain Model}

2.1. Vehicle Layout. The studied topology is a full parallel hybrid electric vehicle shown in Figure 2. ICE and EM are connected to transmission via a single shaft; thus, the ICE and EM speed profile for a given driving cycle are directly given by the driving cycle speed and transmission ratio. As a sequence, the only degree of freedom is choice of the demand torque split decided by EMS. This layout makes possible different operating condition including purely electric, engine alone, hybrid traction, recharge, and combined regeneration and mechanical braking. It is assumed that ICE is turned off when not used and that it does not get cold and loose efficiency during shorter periods of nonuse. Vehicle parameters are listed in Table 1. A backward quasistatic approach has been adapted for modeling powertrain.

2.2. Vehicle Dynamics. Vehicle longitudinal dynamic and the battery state of charge are the only dynamics modeled. All the other components are only algebraic relation or quasistatic models. Vehicle dynamic is based on a base mass plus variable mass of ICE, EM, and battery which all depend on specific component size. Driving cycle speed and acceleration are input to the model. Therefore, tire traction force will be [2]

$$
F_{x}=F_{t}-F_{b}=\underbrace{\frac{1}{2} \rho A_{f} C_{d} v^{2}+m_{v} g \mu_{r}}_{F_{\text {res }}}+\underbrace{m_{v} \dot{v}}_{F_{i}} .
$$

Applying drive train kinematic and lumped efficiencies, demand torque $T_{d}$, and rotational speed $\omega_{d}$ on the right side of the EM will be

$$
\begin{aligned}
& T_{d}=F_{x} \cdot R_{t} \cdot \eta \cdot r(\gamma), \omega_{d}=\frac{v}{r(\gamma) \cdot R_{t}}, \\
& \eta= \begin{cases}\frac{1}{\eta_{g}}, & \text { Driving, } \\
\eta_{g} \cdot \eta_{\mathrm{reg}}, & \text { Braking. }\end{cases}
\end{aligned}
$$




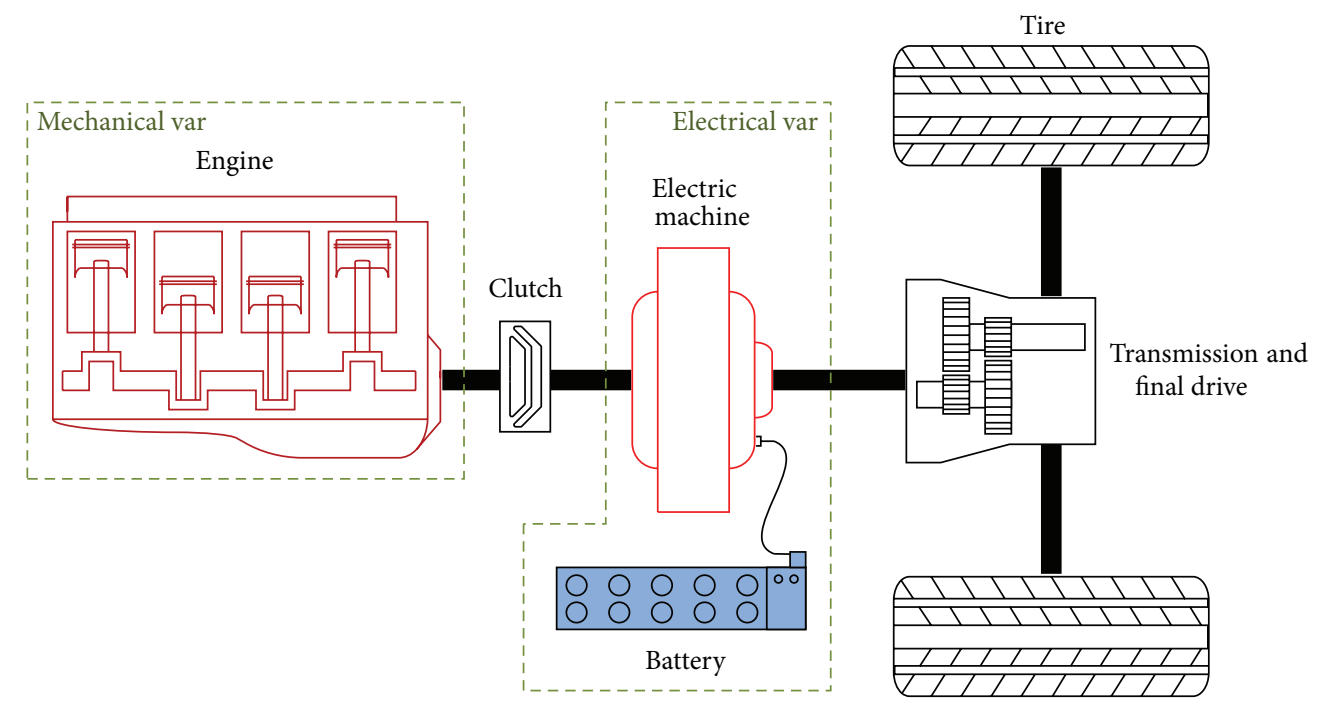

FIGURE 2: Schematic view of the considered hybrid electric vehicle topology.

TABLE 1: Vehicle parameters.

\begin{tabular}{lcc}
\hline Parameter & Description & Value \\
\hline$\rho$ & Air density & $1.202\left[\mathrm{~kg} / \mathrm{m}^{3}\right]$ \\
$C_{d}$ & Aerodynamic drag coefficient & $0.32[-]$ \\
$A_{f}$ & Effective frontal area & $2.13\left[\mathrm{~m}^{2}\right]$ \\
$R_{t}$ & Dynamic tire and wheel radius & $0.282[\mathrm{~m}]$ \\
$\mu_{r}$ & Rolling resistance coefficient & $0.015[-]$ \\
$m_{v 0}$ & Vehicle base mass & $1000[\mathrm{~kg}]$ \\
$r(\gamma)$ & Transmission ratio & $r(\gamma)=\{1.34,1.05,0.78,0.53,0.28\}$ \\
$\eta_{g}$ & Transmission efficiency & $0.95[-]$ \\
$\eta_{\text {reg }}$ & Regeneration efficiency & $0.3[-]$ \\
\hline
\end{tabular}

To simulating driver behavior, a simple shifting strategy shown in Figure 3 is used to choose the gear number $\gamma$. Transmission is modeled as a constant-efficiency converter. An average efficiency was used to include variety of regeneration efficiency in different circumstances.

2.3. Torque Split Factor. The torque split factor $u \in\left[\begin{array}{ll}-1 & 1\end{array}\right]$ used in EMS determines the EM torque $T_{\mathrm{em}}$ using a linear interpolation between three distinct values of $\left.T_{\mathrm{em}}\right|_{\mathcal{U}=-1}$, $\left.T_{\mathrm{em}}\right|_{\mathcal{u}=0}$, and $\left.T_{\mathrm{em}}\right|_{u=1}$ according to Table 2. This is done in order to guarantee a dimensionless control value independent of component peak torque and to minimize numerical problems when using dynamic programming [3]. EM speed $\omega_{\text {em }}$ equals drivetrain demand speed. The combustion engine torque and speed are then given by

$$
T_{\text {ice }}=\left\{\begin{array}{ll}
T_{d}-T_{\text {em }}, & T_{d}>0, \\
0, & T_{d}<0,
\end{array} \quad \omega_{\text {ice }}=\omega_{d},\right.
$$

where $T_{\text {ice }}$ and $\omega_{\text {ice }}$ stand for the engine torque and rotational speed.

2.4. Internal Combustion Engine. A quasistatic method is used for the modeling purpose of the engine. The fuel mass

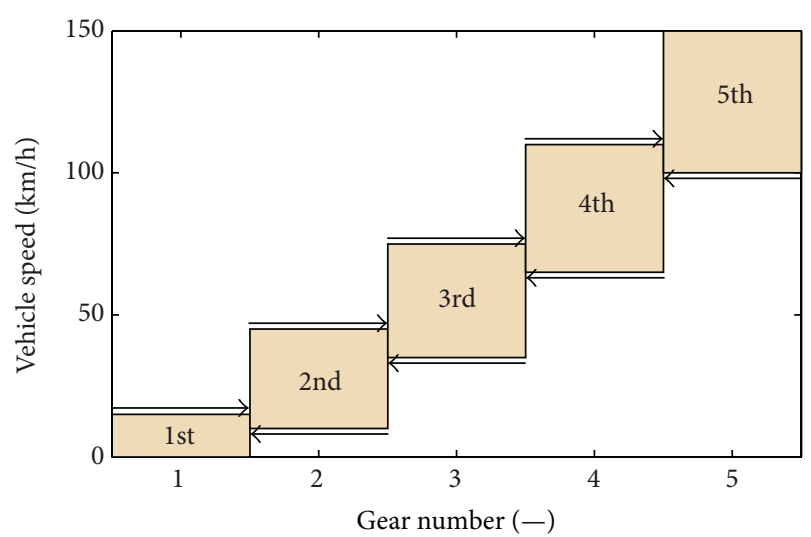

FIGURE 3: Transmission shifting strategy.

flow rate of the naturally aspirating ICE is determined via engine maps of the form

$$
\dot{m}_{f}=f_{\text {ice }}\left(T_{\text {ice }}, \omega_{\text {ice }}\right) .
$$

This map is shown in Figure 4 and is from ADVISOR library [12]. Further, the engine torque satisfies speed 
TABLE 2: Control value definition.

\begin{tabular}{lcccc}
\hline Control & $T_{\mathrm{em}}$ & $T_{\mathrm{ice}}$ & $T_{d}$ & Vehicle operation mode \\
\hline \multirow{2}{*}{$u=-1$} & - & + & + & Maximum recharge \\
& 0 & 0 & - & Conventional braking \\
\hline \multirow{2}{*}{$u \in(-1,0)$} & - & + & + & Partial recharging \\
& 0 & 0 & - & Conventional braking \\
\hline \multirow{2}{*}{$u=0$} & 0 & + & + & Provide all torque by ICE \\
& 0 & 0 & - & Conventional braking \\
\hline \multirow{2}{*}{$u \in(0,1)$} & + & + & + & Provide all torque by ICE and EM \\
& - & 0 & - & Partial regenerative braking \\
\hline \multirow{2}{*}{$u=1$} & + & 0 & + & Provide all torque by EM \\
& - & 0 & - & Maximum regenerative braking \\
\hline
\end{tabular}

dependent inequality $T_{\text {ice }}\left(\omega_{\text {ice }}\right) \leq T_{\text {ice }}^{\max }\left(\omega_{\text {ice }}\right)$. For the optimization purpose, engine maps (torque axis), engine mass, and inequality constraint are scaled linearly with respect to maximum power.

2.5. Electric Machine. A permanent magnet dc machine is used as the secondary mover in full parallel hybrid electric vehicle. EM power input or output to the battery is determined via efficiency map of the form

$$
\eta_{\mathrm{em}}=f_{\mathrm{em}}\left(T_{\mathrm{em}}, \omega_{\mathrm{em}}\right) .
$$

This map is shown in Figure 4 and Adapted from ADVISOR library [12]

$$
P_{\text {batt }}= \begin{cases}\frac{T_{\mathrm{em}} \cdot \omega_{\mathrm{em}}}{\eta_{\mathrm{em}}}, & T_{\mathrm{em}}>0 \\ \eta_{\mathrm{em}} \cdot T_{\mathrm{em}} \cdot \omega_{\mathrm{em}}, & T_{\mathrm{em}}<0\end{cases}
$$

Further, the EM torque satisfies speed dependent inequality $T_{\mathrm{em}}^{\mathrm{min}}\left(\omega_{\mathrm{em}}\right) \leq T_{\mathrm{em}}\left(\omega_{\mathrm{em}}\right) \leq T_{\mathrm{em}}^{\max }\left(\omega_{\mathrm{em}}\right)$. EM map (torque axis), mass, and inequality constraint are scaled linearly with respect to maximum power.

2.6. Battery. The battery model is approximated by an ideal open-circuit voltage source in series with an internal resistance. Therefore, the battery input/output power is the total power supplied to (or by) the EM. The battery current $I_{\text {batt }}$ is then calculated using

$$
I_{\text {Batt }}\left(P_{\text {batt }}\right)=\frac{V_{\mathrm{oc}}-\sqrt{V_{\mathrm{oc}}^{2}-4 \cdot R_{\mathrm{int}} \cdot P_{\mathrm{batt}}}}{2 R_{\mathrm{int}}},
$$

where the battery open circuit voltage $V_{\text {oc }}$ and the battery internal resistance $R_{\text {int }}$ are a nonlinear function of the state of charge $s$ and dependent on the number of cells in series. The battery power is limited to $P_{\text {batt }}^{\min } \leq P_{\text {Batt }} \leq P_{\text {batt }}^{\max }$. The battery's state of charge $s$ is calculated using

$$
\dot{s}=\frac{-I_{\text {Batt }} \eta_{\text {batt }}\left(I_{\text {Batt }}\right)}{Q_{\text {batt }}},
$$

where $Q_{\text {batt }}$ is the battery capacity; further, the battery columbic efficiency is $\eta_{\text {batt }}=0.95$ if charging and otherwise $\eta_{\text {batt }}=1$. For minimizing the risk of premature ageing, the state of charge is usually bounded $s_{\min } \leq s \leq s_{\max }$. The battery data is from ADVISOR library [12].

2.6.1. Battery Scaling. A battery pack can be simply scaled according to its number of cells and the cell capacity. In order to have constant nominal voltage for the battery pack, it is reasonable to choose cell capacity as the only design variable

$$
s f=\frac{Q_{\text {Scaled }}}{Q_{\text {Baseline }}} .
$$

Further, it is assumed that the battery pack has a constant power-to-capacity ratio [10], and therefore the battery pack power limits are proportional to the scale factor $s f$, while the pack voltage remains the same. Also, the battery internal resistance changes as its capacity scale is modified

$$
\begin{gathered}
P_{\text {Scaled }}^{\text {limit }}=s f \cdot P_{\text {Baseline }}^{\text {limit }}, \\
R_{\text {Scaled }}=\frac{R_{\text {Baseline }}}{s f} .
\end{gathered}
$$

2.7. HR. Hybridization ratio in parallel HEVs is defined as below

$$
\mathrm{HR}=\frac{P_{\mathrm{EM}}^{\max }}{P_{\mathrm{EM}}^{\max }+P_{\mathrm{ICE}}^{\max }} .
$$

\section{Energy Management Problem}

There are different operating modes possible for the hybrid electric vehicle. Energy management strategy is used to choose the suitable operating mode and corresponding power torque split between sources. This task is accomplished by utilizing functional or online criteria (fuel consumption and emission) as the performance index. Since design study is of interest in this paper, an optimal method should be employed for the energy management and determination of the FC. In fact, design study of the vehicle needs the ultimate potential of the efficiency improvements for every set of design variables. Furthermore, charge sustenance should be accomplished because equality of the initial and final state value eliminates effect of the electrical energy usage on fuel economy and makes it possible to have fair comparisons between different sizes of the energy storage system (battery). Therefore, a powerful numerical method, deterministic dynamic programming with final state constraint, is used in this paper to serve as the energy management strategy.

3.1. Optimal Control Problem. The optimal control problem under study consists in minimizing the FC of the vehicle along a prescribed vehicle cycle, taking into account physical 


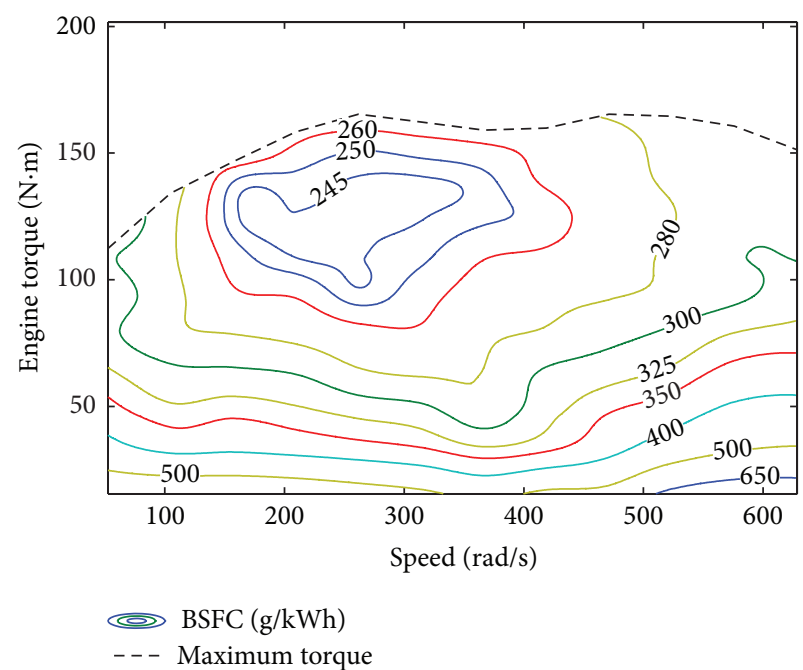

(a)

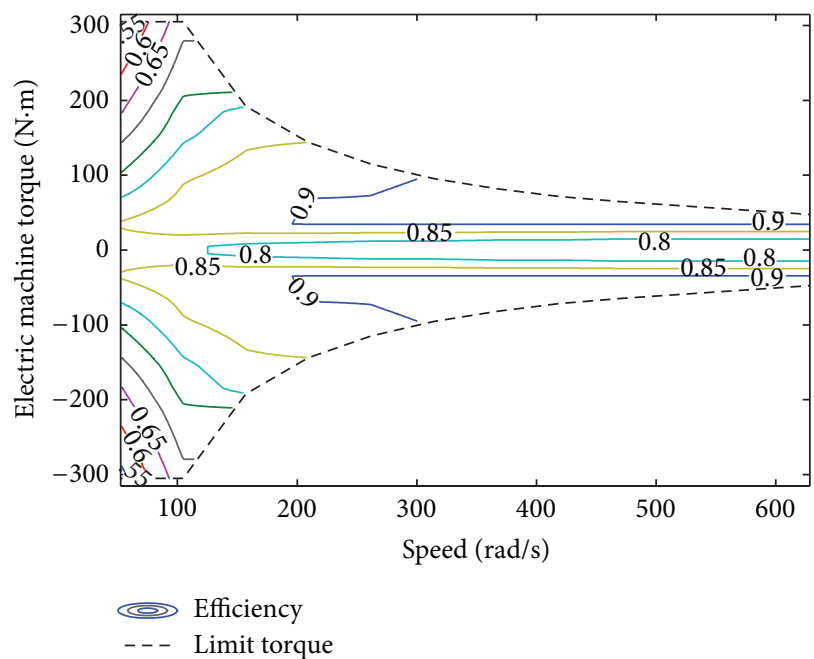

(b)

FIGURE 4: ICE brake specific fuel consumption (a) and EM efficiency (b) both as a function of rotational speed and torque.

constraints from battery, ICE, and EM. The general optimization problem is as follows:

$\min$

$$
J(u):=\int_{0}^{t_{f}} L(x(t), u(t), t) d t
$$

subject to: $\dot{x}(t)=f(x(t), u(t), t)$,

$$
\begin{aligned}
& x(0)=x_{0}, \\
& x\left(t_{f}\right)=x_{f} \\
& x(t) \in\left[X_{\text {min }}, X_{\max }\right] \\
& u(t) \in\left[U_{\min }, U_{\max }\right],
\end{aligned}
$$

where 0 and $t_{f}$ are, respectively, initial and the final times of the prescribed driving cycle, $u(t)$ is the torque split factor, $x(t)$ is the battery state of charge, $L(x(t), u(t), t)$ is the instantaneous FC, and $f(x(t), u(t), t)$ is the function that controls the variation of the battery state of charge dynamic.

It should be noticed that usually a terminal cost is added to the accumulative cost function for penalizing final state deviation. But this method of treating final state deviation is not interesting in optimal sizing of HEVs. Whatever the battery capacity is, a small deterioration of the charge sustaining may result in unfair comparisons of different sizes, and therefore a modified version of the basic DP is developed to induce hard constraint on final state.

3.2. DP Algorithm. This section gives a brief overview of the DP algorithm used in this paper for optimal control and thus design study. Two key features of the DP are an underlying discrete-time dynamic system and a cost function that is additive over time. Therefore, using (8), the discretizedmodel should be employed as follows:

$$
x_{k+1}=x_{k}-f_{k}\left(x_{k}, u_{k}, w_{k}\right), \quad k=0, \ldots, N, N=\frac{t_{f}}{\Delta T},
$$

where $k$ indexes discrete time, $x_{k}$ is the state of the system, $u_{k}$ is the control value to be selected at time $k, w_{k}$ is the disturbance, and $N$ is horizon. The cost function is additive in the sense that the cost incurred at time $k$, denoted by $L_{k}\left(x_{k}, \mu_{k}, w_{k}\right)$, accumulates over time, and thus the total cost is

$$
\sum_{k=0}^{N-1} L_{k}\left(x_{k}, \mu_{k}, w_{k}\right)
$$

Now consider a control policy $\pi$ as a sequence $\left\{\mu_{0}, \mu_{2}, \ldots, \mu_{N-1}\right\}$, and then the corresponding cost for a fixed initial state $x_{0}$ is

$$
j_{\pi}\left(x_{0}\right)=\sum_{k=0}^{N-1} L_{k}\left(x_{k}, u_{k}, w_{k}\right) .
$$

For a given initial state $x_{0}$, an optimal policy $\pi^{*}$ is the one that minimizes above cost

$$
j_{\pi^{*}}\left(x_{0}\right)=\min _{\pi \in \Pi} j_{\pi}\left(x_{0}\right) .
$$

In the above equation, $\Pi$ stands for the set of all admissible policies.

Consider the proposed problem and the following steps for determining the optimal policy and thus optimal cost.

3.2.1. Exclusion of the Infeasible States (Boundary-Line Method). Optimal control problem formulated in this study contains state equality constraint at final time (terminal 


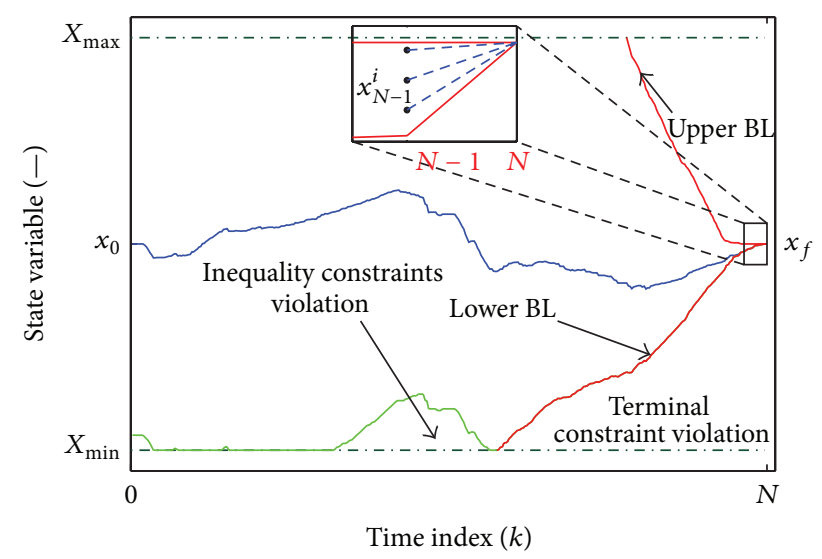

FIGURE 5: Schematic of an optimal trajectory solved by DP and the corresponding infeasible areas.

constraint) and state inequality constraints over the time horizon (12). As a consequence, any value of the state over the time horizon of the problem, which causes the trajectory of the system violates mentioned constraints, is regarded as the infeasible state and should be excluded from state-time space.

Terminal Constraint. Boundary-Line (BL) DP was first introduced in [13] to overcome reachability of the partially fixed final state. This method is modified in this paper to satisfy fully fixed final state. Mentioned method evaluates extreme achievable value of the state governed by the inverse model starting from final time and sequentially going backward as many time steps as the inequality constraints are not active. As a result, two boundary lines are calculated (Figure 5). Excluding the subset of space which is outside the upper and lower boundary lines enforces trajectories to reach final state.

Inequality Constraints. Any value of the state over the time horizon of the problem which may result in a trajectory violating inequality constraints should be omitted. Unfortunately, it is not possible to estimate them priorly. Therefore, exclusion of mentioned states is integrated in the backward step of the DP.

In this way, feasible state values for every time index $\left(X_{k}\right)$ are determined and will be saved for further usages.

3.2.2. Backward in Time (Optimal Map). For numerical implementation of the DP, the state and control should be discretized considering feasible values of states calculated priory. The dynamic programming technique rests on a very simple idea, the principle of optimality. It suggests that an optimal policy can be constructed in piecemeal fashion, first constructing an optimal policy for the "tail-subproblem" involving last stages and then extending the optimal policy to the "tail-subproblem" involving last two stage and proceed toward last $N$ stage to include the entire problem. As a consequence, an optimal cost-to-go function $j_{k}\left(x^{i}\right)$ should be introduced and used for the "tail subproblem." This function saves optimal accumulative cost for the state $x^{i}$ at time $k$ for getting to the final state $x_{f}$. Thus, the optimal map is generated in two steps as follows.

Step $1(k=N-1)$. Application of a boundary line method together with a fixed final state cancel searching for optimal control values at prefinal step because there is a unique control value corresponding to departure to the final state. That is,

$$
\begin{array}{ll}
\text { find } & u_{N-1}^{i} \\
\text { subject to: } & x_{N}-x_{N-1}^{i}+f\left(x_{N-1}^{i}, u_{N-1}, w_{N-1}\right)=0,
\end{array}
$$

where $u_{k}^{i}$ refers to the optimal control value at time $k$ and for the state $x_{k}^{i}$. Further, optimal cost-to-function should be initiated

$$
j_{N-1}\left(x^{i}\right)=L_{N-1}\left(x_{N-1}^{i}, u_{N-1}^{i}, w_{k}\right) .
$$

Now, we are assured that every trajectory of the system at time $k=N-1$ will depart to the $x_{N}=x_{f}$.

Step 2 ( $k=N-2$ to 0$)$. Based on principles of optimality [14], the optimal cost-to-go function $j_{k}\left(x^{i}\right)$ at every node in discretized state-space should be evaluated by proceeding backward in time:

$$
\begin{array}{r}
{\left[j_{k}\left(x^{i}\right), u_{k}^{i}\right]=\min \left\{L_{k}\left(x_{k}^{i}, u\right)+j_{k+1}\left(x_{k+1}\right)\right\},} \\
u \in U, \\
x_{k+1}=x_{k}^{i}-f_{k}\left(x_{k}^{i}, u, w_{k}\right) .
\end{array}
$$

The optimal control and cost-to-go function is given by the argument that minimizes the right-hand side of (19).

As stated in previous section (inequality constraints), application of the control values, $u \in U$ at $x_{k}^{i}$, in system dynamic may result in $x_{k+1}$ which does not belong to the feasible states of the proceeding time index $\left(X_{k+1}\right)$, and therefore the corresponding state node should be omitted from the set of feasible states of the current time index. This infeasibility is shown in Figure 5 under the name "inequality constraints violation."

The cost-to-go function used in (19) is evaluated only on discretized points in the state space while the output of the model function $x_{k+1}$ is a continuous variable in the state space which may do not coincide with the nodes of the state grid. Therefore, an appropriate interpolation scheme should be used.

3.2.3. Forwards Dynamics. Given initial state value $x_{0}$ and solving system dynamic using optimal control map $u_{k}^{i}$ obtained in (19), one can generate the optimal trajectory and evaluate the corresponding cost.

3.3. Validation. To validate vehicle model, ADVISOR software is used and a medium-size vehicle based on data represented in this paper has been constructed. There are some differences between these two models but fortunately the result of fuel economy is in an acceptable tolerance. 
TABLE 3: Nominal parameters of HEV.

\begin{tabular}{llr}
\hline Component & & Specification \\
\hline & Peak torque/power & $165-\mathrm{Nm} / 95-\mathrm{kW}$ \\
ICE (1.9 [1] DOHC SI engine) & Optimum consumption & $3.2 \mathrm{~kg} / \mathrm{kW}$ \\
& Power density & $300-\mathrm{Nm} / 30-\mathrm{kW}$ \\
& Peak torque/power & $91 \%$ \\
EM (PMDC) & Peak efficiency & $3 \mathrm{~kg} / \mathrm{kW}$ \\
& Power density (with accessories) & $12 \mathrm{Ah}$ \\
Battery pack (Saft lithium-ion battery) & Capacity & $120 \mathrm{Wh} / \mathrm{kg}$ \\
& Power density & $2.81 \mathrm{~kW} / \mathrm{Ah}$ \\
\hline
\end{tabular}

Unfortunately, the validation of optimal energy management strategy is not a straight forward process, because traditional software only supports on-line strategies.

In this section, the nominal parameters of an $\mathrm{HEV}$ are used for validation dynamic programming algorithm and corresponding optimal energy management problem. It is important to be assured about accuracy of the algorithm, because fuel consumption of the vehicle is used as the criteria for sizing design study.

In order to investigate performance of the proposed strategy, vehicle is driven along the 3rd class of WLTC (world harmonized test cycle) and corresponding measures are evaluated. The nominal parameters of a case study presented in Table 3. Furthermore, three values of the battery capacity $\{12,24,36\} A h$ are used for the evaluation of the optimal trajectories. Initial value of the state of charge is 0.5 and the same value is expected at final time. For practical consideration, the SOC is bounded between 0.3 and 0.7. In the case of DP setting, control input grid is chosen to be 50 . The number of elements in the state grid is 300 and the time step is chosen to be unit. Generally, two major tasks were considered for the designed energy management strategy.

At first, it is expected that the battery initial and final states of charge are identical for different values of the capacity. As shown in Figure 6, the proposed algorithm is charge sustain in nature independent of the problem parameters. Thus, the electric storage system (battery) is used only as an energy buffer and the vehicle propelling energy is globally provided by fuel.

Secondly, reduction of the FC occurs as a result of the efficiency improvement. Therefore, the proposed energy management strategy should try the best to increase efficiency. Concluded from operating diagram of the ICE, Figure 7, most of the operating points have clearly migrated towards higher efficiency areas. It should be noticed that dictated engine speed and electric path efficiency prevent from shifting rest of the operating points. This task is fulfilled by optimal selection of the operation mode (Figure 8) and torque split. Detailed simulation data is shown in Figure 9.

The developed optimal method can accomplish expected tasks, whatever the powertrain size and the driving cycle are. In fact, influences of the control strategy on the fuel economy are eliminated and it is possible to focus on the design study.

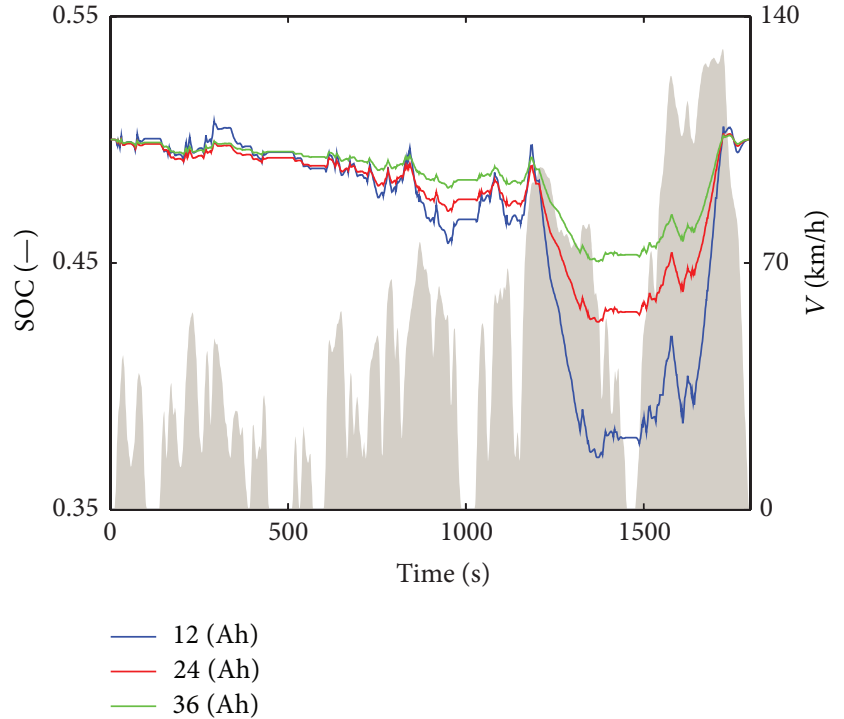

FIGURE 6: Battery optimal state of charge trajectory and corresponding WLTC-3 driving cycle profile.

\section{Design Study}

The design study is an important step in design-optimization process, which gives a good insight to problem and effects of different parameters on system criteria. This paper investigates only the sizing problem in the design study and investigations on topology and transmission are left for future research. Sizing problem of the HEVs generally includes three design variables, peak power of the ICE, EM, and capacity of the battery.

In this study, the general form of mentioned problem was reduced to two-variable design problem and considers only power sources (ICE and EM). Structure of the HEVs enables us to simply set battery capacity for a defined pair of the EM and ICE peak power.

According to powertrain structure of the HEV, EM power is always supplied by the battery pack, and thus the limit power of battery pack should correspond to the EM peak power demand on the driver side (motoric and generator mode). Therefore, the capacity of the battery is then given by 


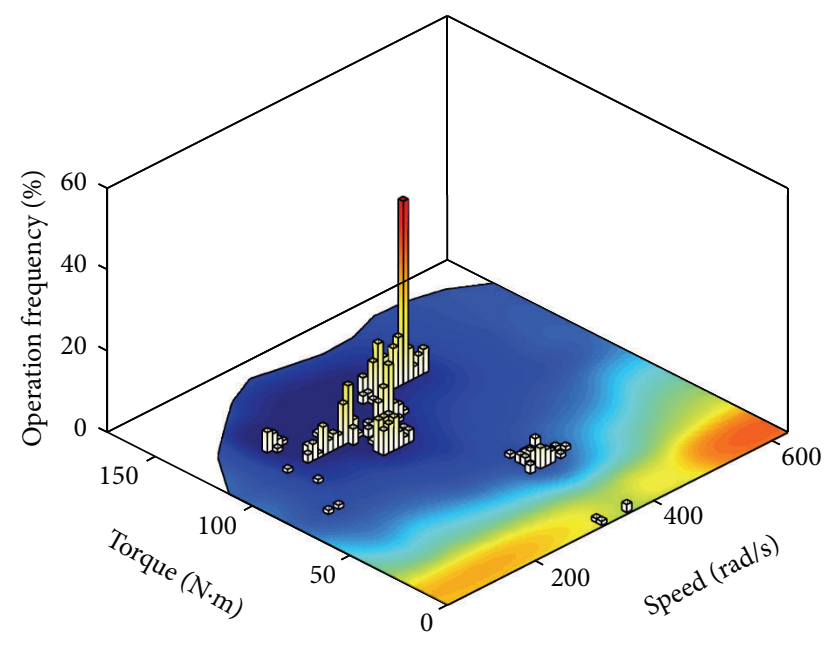

FIGURE 7: Engine operation histogram and efficiency map (on bottom) during WLTC-3.

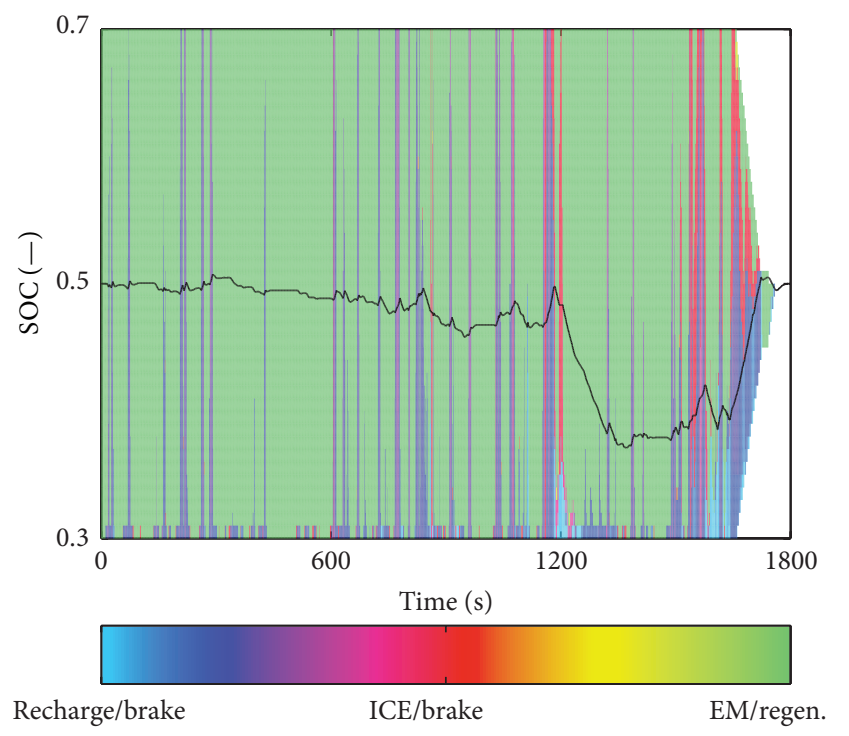

FIGURE 8: Optimal control signal map during WLTC-3.

the peak power of the EM on the driver side, together with the constant maximum power-to-capacity ratio of the battery pack.

Effects of different components size on vehicle aggregate mass are an important aspect of sizing. In this paper, a linear mass-power scaling factor was adopted.

4.1. Component Size. In this section, a DOE method has been scheduled with design variables varying from 0 to $100[\mathrm{~kW}]$ with a step of $5[\mathrm{~kW}]$ for the ICE and EM peak power. Different characteristics of the desired components are generated by suitable scaling of baseline data (see Table 3). Of course, the proposed optimal energy management strategy is used to compute the minimum FC for every pair of design variables.

4.2. Driving Cycles. Unfourtunately, the proceeding methodology for minimization of the FC is cycle oriented. Therefore, a comparison between different driving cycles is made in Figure 10, and suitable driving cycles resembeling a variety of driving conditions are choosen. Four driving cycles have been considerd to estimate the fuel economy. NEDC (New European Driving Cycle) is used as benchmark to estimate FC in Europe. WLTC, US06, and FTP-75 are the other driving cycles derived from real speed mesurement.

\section{Result and Discussion}

The fuel economy maps regarding the proposed method are shown in Figure 11 for the mentioned driving cycles. These maps are constructed by drawing isoconsumption contours. The horizontal and vertical axes are, respectively, corresponding to pure electric $\left(P_{\mathrm{EM}}=0\right)$ and pure ICE vehicle $\left(P_{\mathrm{ICE}}=0\right)$. The pure conventional vehicle can be used as a benchmark measure for fuel economy. The constant power-to-weight $(p / w)$ lines are used to reflect different levels of acceleration performance. The "infeasible area" represents the undersized ICE and EM, which cannot fulfill the driving cycle either torque demand (left border) or energy demand (right border).

Based on previous argue, the sizing problem naturally consists of two design variables related to mechanical part and electrical part. By inspecting performance constraint and characteristics of specified sizing problem, these two variables can be reduced to one as follows.

Clearly, there is an approximate trade-off between fuel economy and drivability. As shown in the figures, the constant $p / w$ lines include different levels of consumption (intersection of consumption contours and constant $p / w$ lines).

Considering HR definition, it is simply proved that the $\mathrm{HR}$ is constant on lines passing through origin, so the HR variable can parameterize constant $p / w$ lines. So $H R$ is a natural selection as design variable (instead of two traditional sizing variables).

To better discuss this concept, consider that, for an optimal sizing of the parallel HEV, it is not necessary to search all the possible pairs of ICE and EM peak power. Considering a fixed drivability performance, the above sizing problem can be reduced to a problem with single design variable.

For example, FC gains (HEV FC/conventional vehicle FC) are illustrated in Figure 12 for different HRs with constant $p / w$ of 85 . Since the range of $p / w$ is varied between different driving cycles, some cycles are modified in a way to be compatible with the mentioned $p / w$.

It is obvious from Figure 12 that fuel economy improvements are not equal between different driving cycles and in different HRs.

At first, regarding HEV topology, the operating speed of ICE and EM is dictated by the speed distributions of the driving cycle and therefore delivered torque by the ICE and EM is the only parameter which can be controlled by optimal 


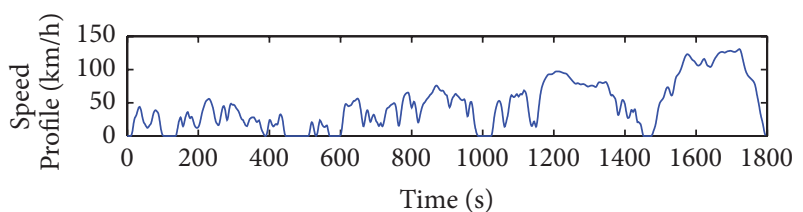

(a)

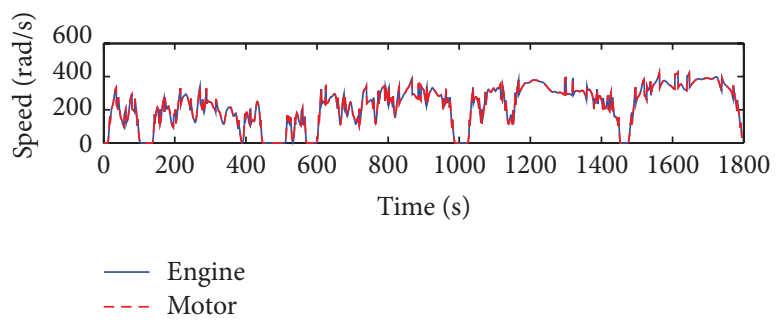

(c)

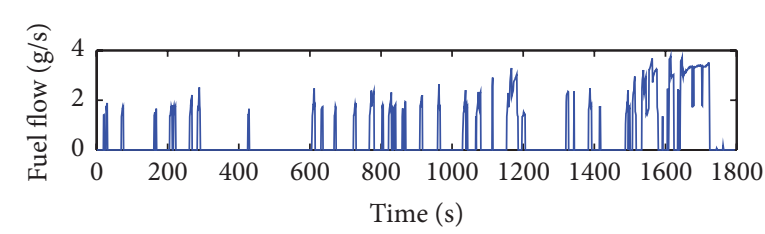

(e)

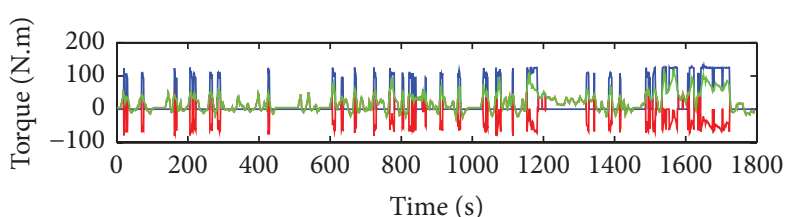

Engine _ Demand

- Motor

(b)

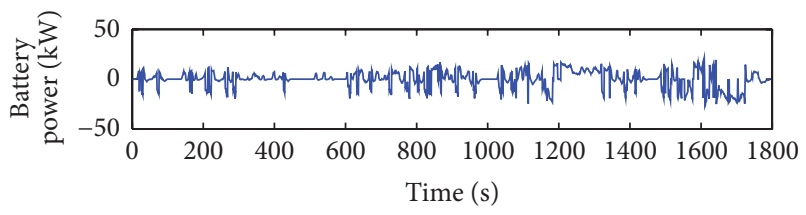

(d)

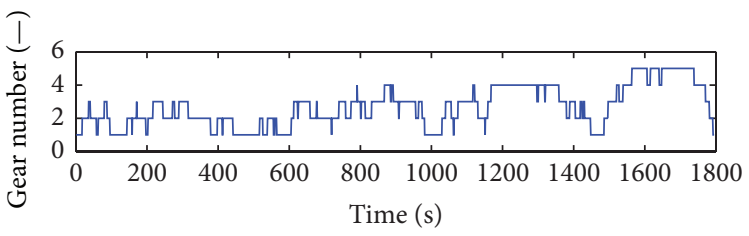

(f)

FIGURE 9: Optimal torque split between ICE (-) and EM (-) in different operation mode during WLTC-3.

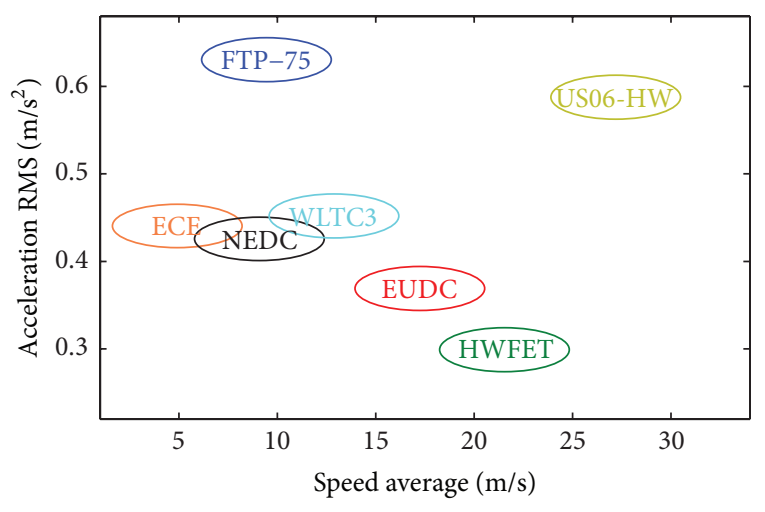

FIGURE 10: RMS characteristics of driving cycles.

control strategy. As a consequence, order of the magnitude of the FC reduction will be different between driving cycles.

Secondly, FC reduction gain possesses a minimum value with respect to HR. The reason behind this behavior is better understood by repartitioning engine operating efficiency and delivered energy. Figure 13 illustrates percentage of the ICE operating at optimal region (BSFC $<260 \mathrm{~g} / \mathrm{kWh}$ ) and nonoptimal region (BSFC $>260 \mathrm{~g} / \mathrm{kWh}$ ).

To better understand sizing results, the fuel consumption is divided into two components: engine operating frequency and efficiency of operating points. These two components form the optimality of solution.

Figure 13 shows these two concepts. The left axis corresponds to ICE efficiency repartition, optimal area (green), and nonoptimal area (yellow), and right axis shows delivered energy by ICE.

Primarily it was concluded from Figure 12 that the optimal HR for the WLTC-3 is around 65\%. The electric component size impacts the control strategy by giving it more or less freedom in shifting the operating points. As a consequence, it seems that the optimal HR corresponds therefore to the necessary size of electric components to move the ICE operating points to optimal areas while upsizing EM enables capturing more regenerative energy, but this is not the case because $25 \%$ of hybridization is enough to shift most of the operating points of the ICE to optimal areas. The reason behind reduced FC gain after $25 \%$ of $\mathrm{HR}$ is the lower amount of the energy delivered by ICE in a driving cycle (increased electric path efficiency). As the HR goes after 65\%, the low efficiency of electrical path makes ICE to give more energy and so increasing FC. Concluded from above, there is an optimal HR which can explore ultimate fuel saving.

Figure 13 emphasizes that the optimal energy management is necessary for optimal sizing and online heuristic strategy cannot tend to optimum sizing; for example, the engine operates almost all in optimum region at $\mathrm{HR}$ around $25 \%$ but this is not the optimal HR. 


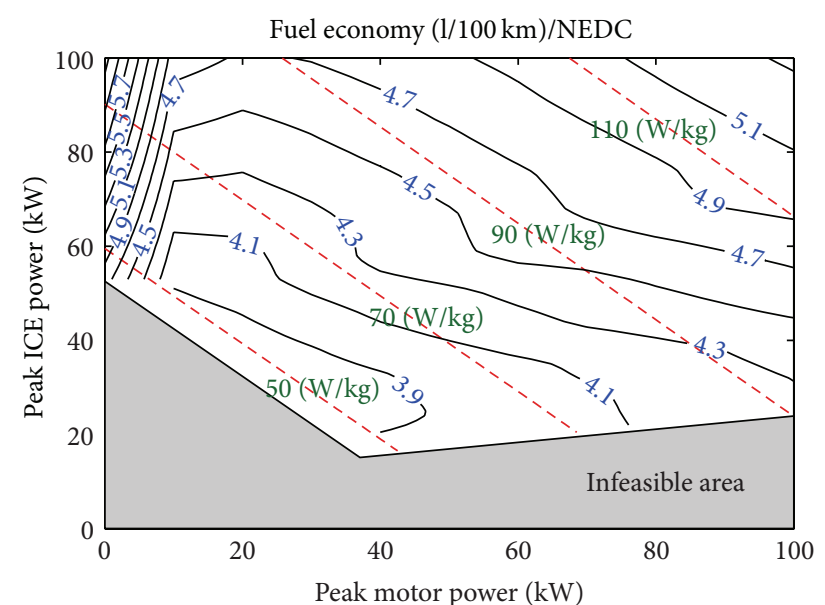

(a)

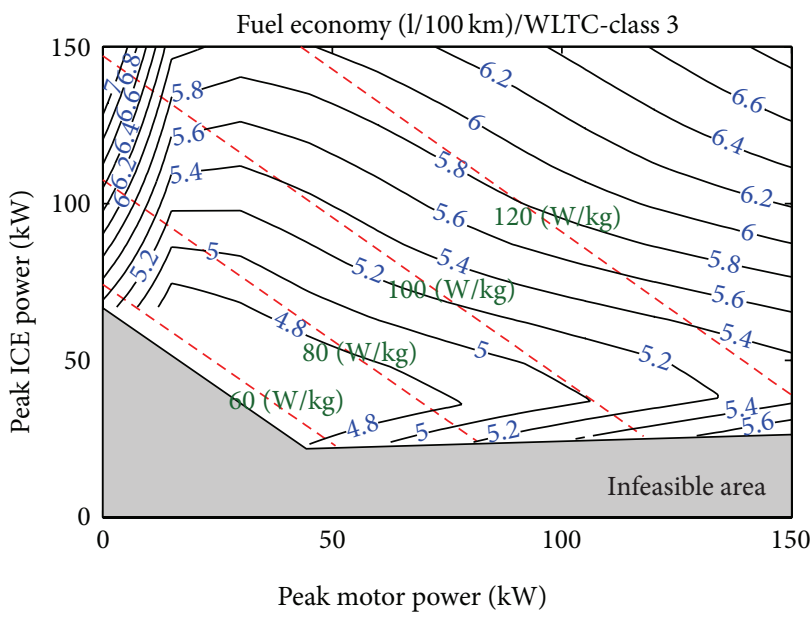

(c)

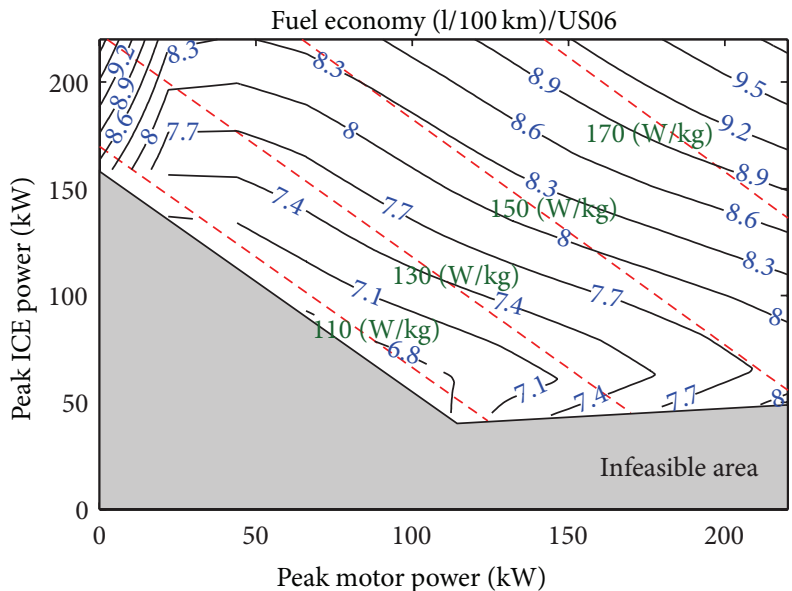

(b)

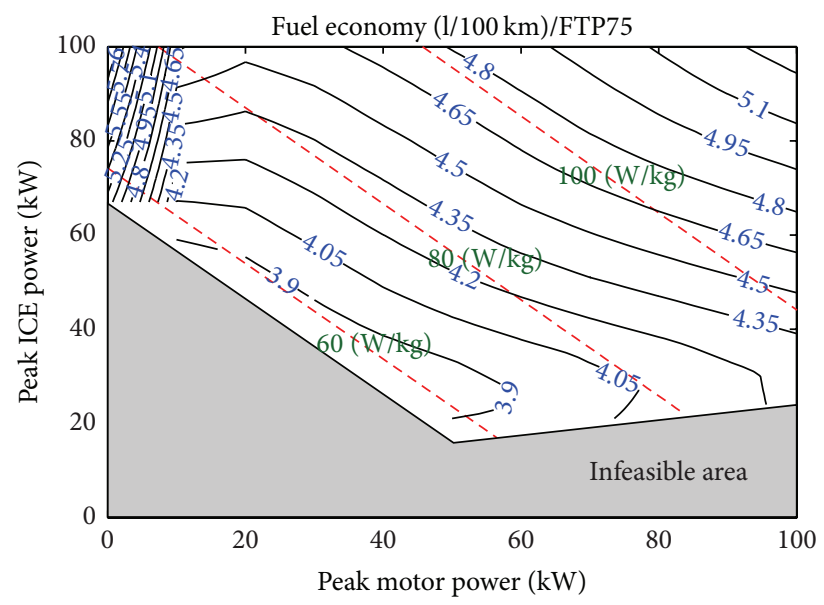

(d)

FIGURE 11: Isoconsumption levels (-) computed on several driving cycles with constant $p / w$ lines (- -).

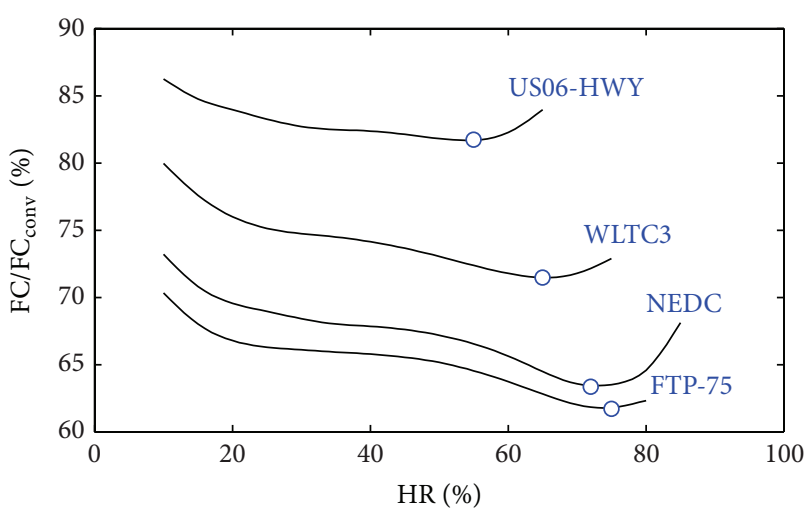

FIgURE 12: Effect of HR on FC gain for constant $p / w$.

\section{Summary and Conclusion}

In the conceptual design phase of a hybrid vehicle, estimation of the vehicle's general attributes is a major aid to the managers and decision-makers. Consider, for example, the

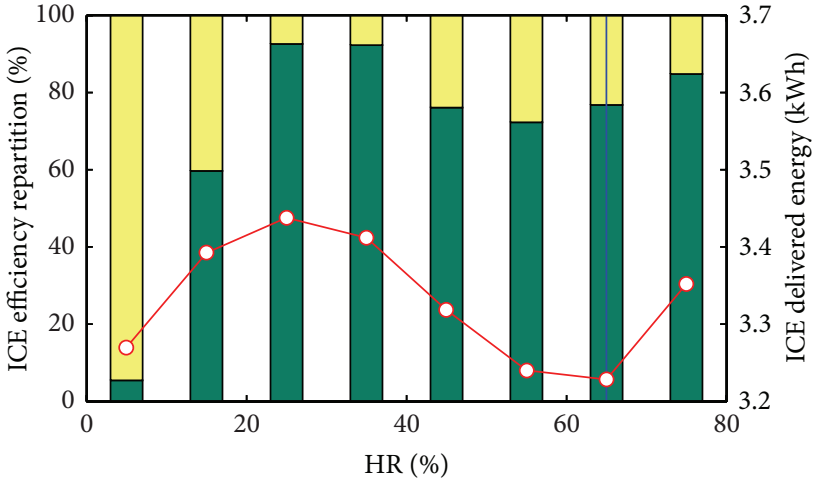

FIGURE 13: ICE efficiency histogram (green for optimal area) and ICE delivered energy on WLTC-3.

plan to convert an existing ICE vehicle into a hybrid one. The managers should make decisions based on various, usually conflicting, criteria including the conversion cost and the economy obtained from hybridization. To make 


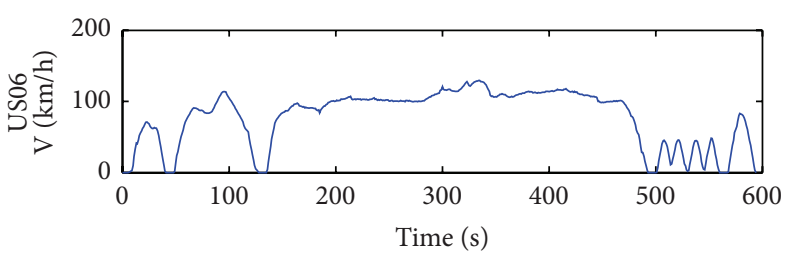

(a)

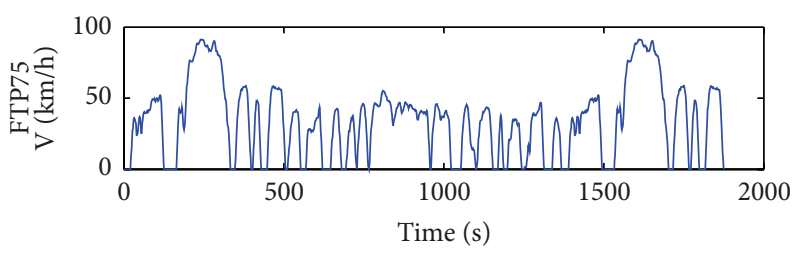

(c)

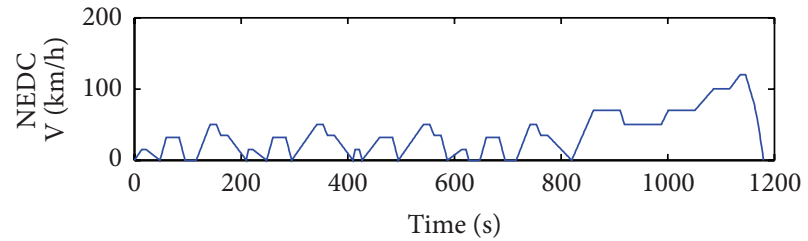

(b)

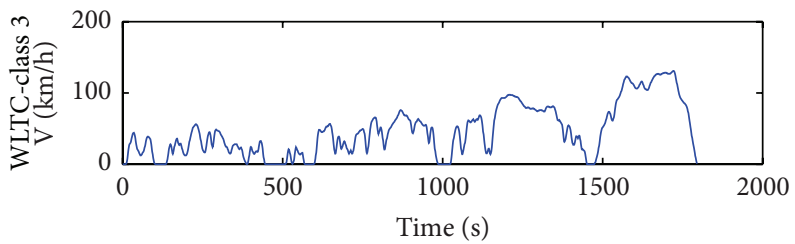

(d)

FIGURE 14: US06, NEDC, FTP75, and WLTC-3 speed versus time.

that decision easier to make, an optimal energy management strategy for parallel HEVs was formulated based on dynamic programming. The strategy takes into account the problem of imbalanced states of charge. To investigate the effects of the power source sizing, vehicle's fuel economy was estimated for various driving conditions. Next, the optimal hybridization ratio satisfying a predefined drivability constraint while maximizing the fuel economy was determined. It was shown that (1) parallel HEV's sizing variables can be reduced to the combination of the HR and drivability constraints; (2) optimal performance requires the downsizing of the ICE, and (3) increasing the hybridization ratio is desired as long as it does not negatively affect the performance of the control strategy. The scope of the present article was limited to the parallel HEV's sizing problem and the optimization of the vehicle's topology and transmission is the subjects of the authors' future work. The developed code and its user interface are placed freely in the address (http://sites.google.com/site/ansarey/) in order to allow researchers use it for future development and generalize the software and its components library [15].

\section{Appendix}

See Figure 14.

\section{Conflict of Interests}

The authors declare that there is no conflict of interests regarding the publication of this paper.

\section{References}

[1] P. Pisu and G. Rizzoni, "A comparative study of supervisory control strategies for hybrid electric vehicles," IEEE Transactions on Control Systems Technology, vol. 15, no. 3, pp. 506-518, 2007.

[2] L. Guzzella, Vehicle Propulsion Systems, Springer, New York, NY, USA, 2005.
[3] O. Sundström, L. Guzzella, and P. Soltic, “Torque-assist hybrid electric powertrain sizing: from optimal control towards a sizing law," IEEE Transactions on Control Systems Technology, vol. 18, no. 4, pp. 837-849, 2010.

[4] L. Thibault, O. Grondin, C. Querel, and G. Corde, "Energy management strategy and optimal hybridization level for a diesel HEV," SAE International Journal of Alternative Powertrains, vol. 1, no. 1, pp. 260-271, 2012.

[5] T. Nüesch, T. Ott, S. Ebbesen, and L. Guzzella, "Cost and fuel-optimal selection of HEV topologies using particle swarm optimization and dynamic programming," in Proceedings of the American Control Conference (ACC '12), pp. 1302-1307, Montreal, Canada, June 2012.

[6] U. Zoelch and D. Schroeder, "Dynamic optimization method for design and rating of the components of a hybrid vehicle," International Journal of Vehicle Design, vol. 19, no. 1, pp. 1-13, 1998.

[7] T. Hofman, S. Ebbesen, and L. Guzzella, "Topology optimization for hybrid electric vehicles with automated transmissions," IEEE Transactions on Vehicular Technology, vol. 61, no. 6, pp. 2442-2451, 2012.

[8] J. Bernard, S. Delprat, F. N. Büchi, and T. M. Guerra, "Fuelcell hybrid powertrain: toward minimization of hydrogen consumption," IEEE Transactions on Vehicular Technology, vol. 58, no. 7, pp. 3168-3176, 2009.

[9] S. M. Lukic and A. Emadi, "Effects of drivetrain hybridization on fuel economy and dynamic performance of parallel hybrid electric vehicles," IEEE Transactions on Vehicular Technology, vol. 53, no. 2, pp. 385-389, 2004.

[10] M.-J. Kim and H. Peng, "Power management and design optimization of fuel cell/battery hybrid vehicles," Journal of Power Sources, vol. 165, no. 2, pp. 819-832, 2007.

[11] H. K. Fathy, J. A. Reyer, P. Y. Papalambros, and A. G. Ulsoy, "On the coupling between the plant and controller optimization problems," in Proceedings of the American Control Conference, June 2001.

[12] K. B. Wipke, M. R. Cuddy, and S. D. Burch, "ADVISOR 2.1: a user-friendly advanced powertrain simulation using a combined backward/forward approach," IEEE Transactions on Vehicular Technology, vol. 48, no. 6, pp. 1751-1761, 1999.

[13] O. Sundström, D. Ambühl, and L. Guzzella, "On implementation of dynamic programming for optimal control problems 
with final state constraints," Oil and Gas Science and Technology, vol. 65, no. 1, pp. 91-102, 2010.

[14] R. E. Bellman, Dynamic Programming, Dover Publications, New York, NY, USA, 2003.

[15] M. Ansarey, M. Shariat Panahi, H. Ziarati, and M. Mahjoob, "Optimal energy management in a dual-storage fuel-cell hybrid vehicle using multi-dimensional dynamic programming," Journal of Power Sources, vol. 250, pp. 359-371, 2014. 


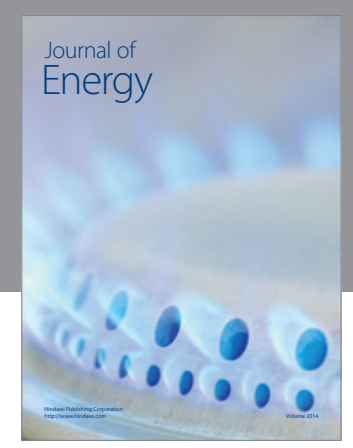

Journal of

Industrial Engineering
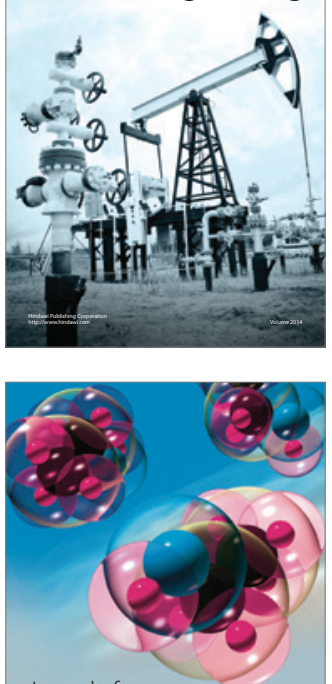

Fuels
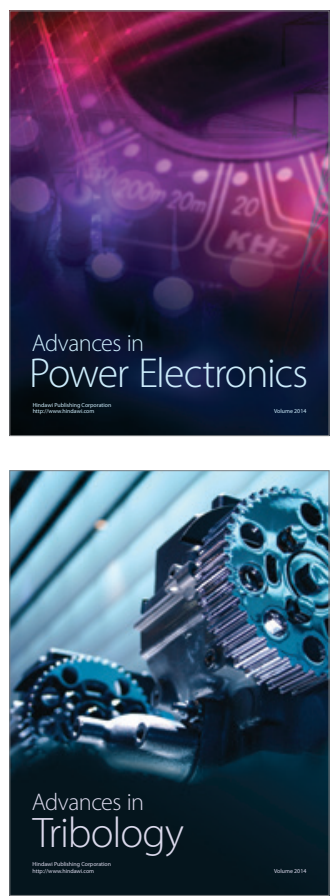

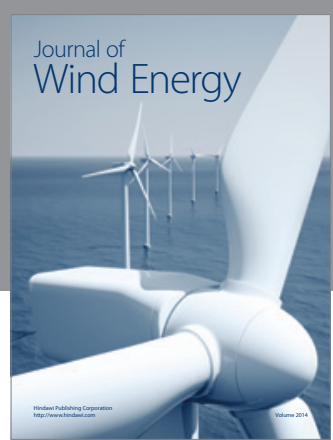

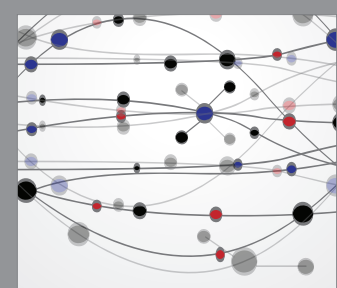

The Scientific World Journal

Submit your manuscripts at http://www.hindawi.com

Journal of

Structures
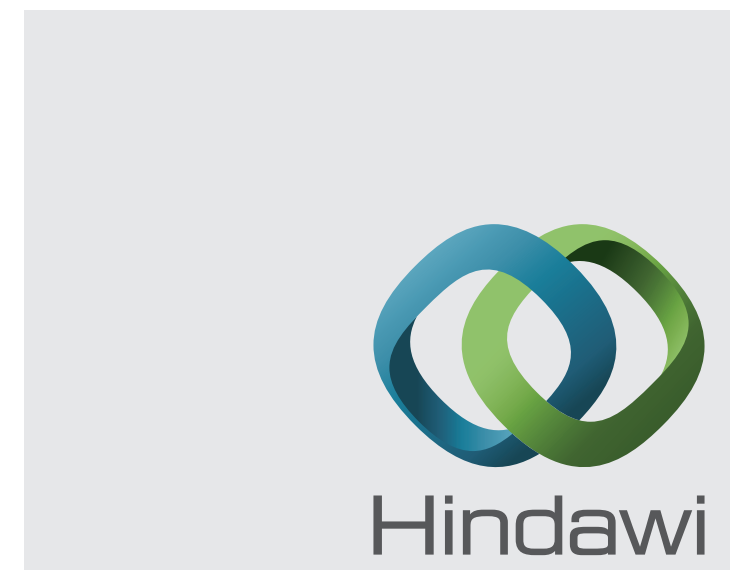

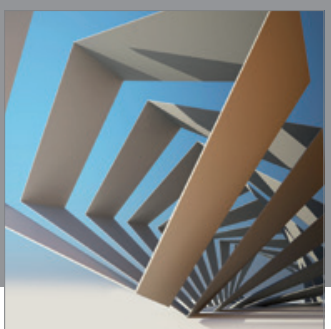

Rotating

Machinery
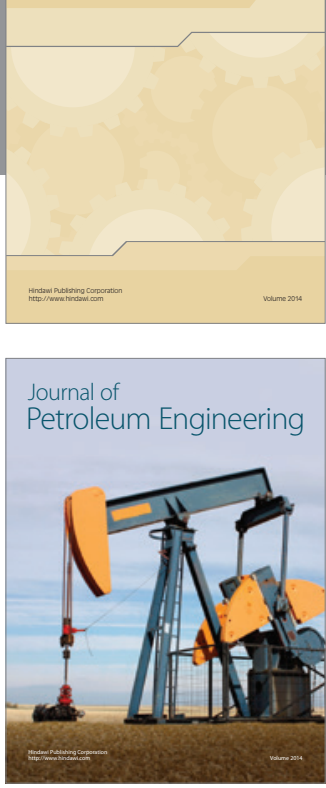

Journal of

Solar Energy
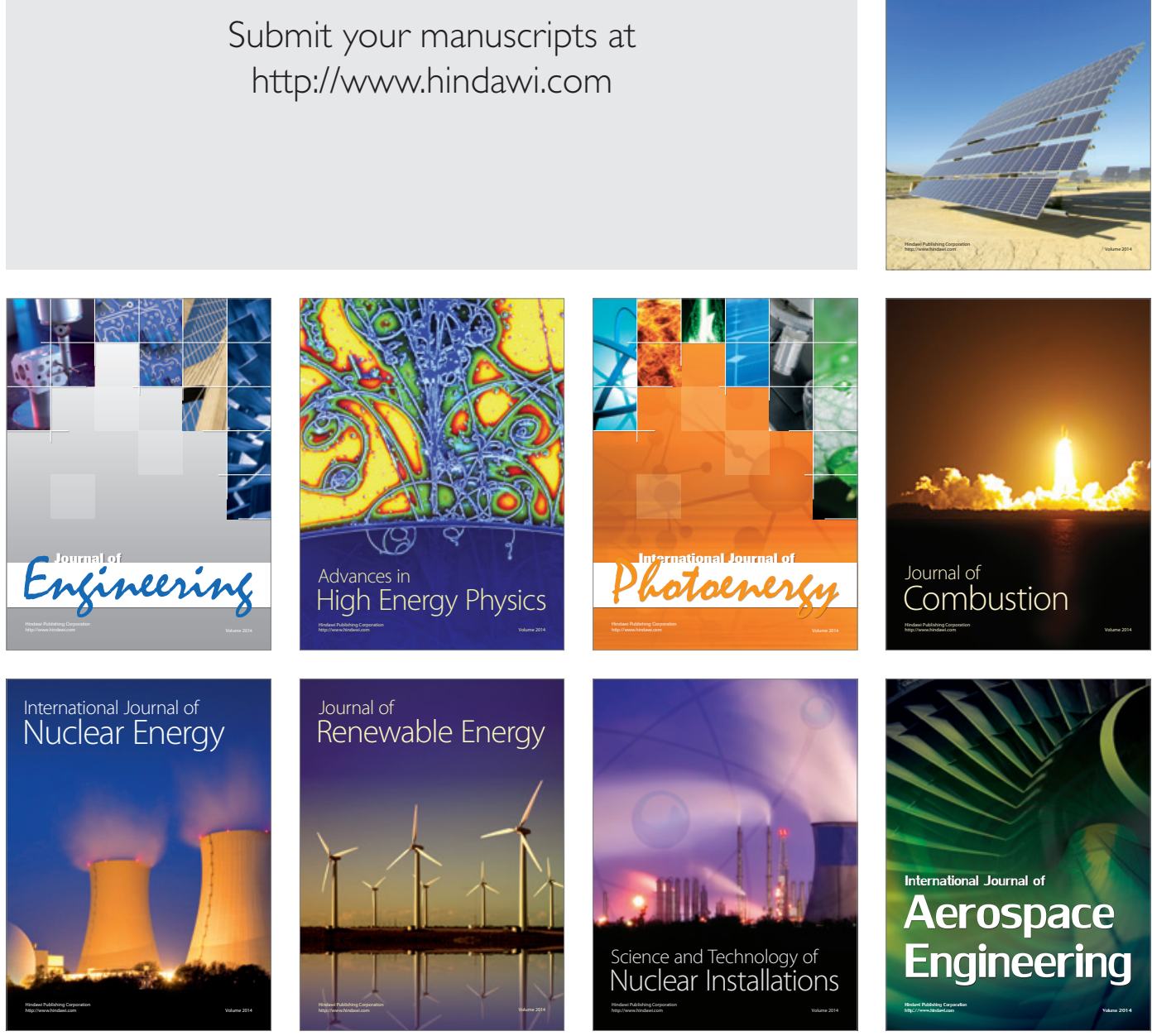